Article

\title{
Fluctuations of Intensive Quantities in Statistical Thermodynamics
}

\author{
Artur E. Ruuge \\ Department of Mathematics and Computer Science, University of Antwerp, \\ Middelheim Campus Building G, Middelheimlaan 1, Antwerp B-2020, Belgium; \\ E-Mail: artur.ruuge@uantwerpen.be; Tel.: +32-3-265-3872; Fax: +32-3-265-3777
}

Received: 17 September 2013; in revised form: 31 October 2013 / Accepted: 6 November 2013 / Published: 11 November 2013

\begin{abstract}
In phenomenological thermodynamics, the canonical coordinates of a physical system split in pairs, with each pair consisting of an extensive quantity and an intensive one. In the present paper, the quasithermodynamic fluctuation theory of a model system of a large number of oscillators is extended to statistical thermodynamics based on the idea of perceiving the fluctuations of intensive variables as the fluctuations of specific extensive ones in a "thermodynamically dual" system. The extension is motivated by the symmetry of the problem in the context of an analogy with quantum mechanics, which is stated in terms of a generalized Pauli problem for the thermodynamic fluctuations. The doubled Boltzmann constant divided by the number of particles plays a similar role as the Planck constant.
\end{abstract}

Keywords: fluctuations; thermodynamics; quantization; Pauli problem; duality

\section{Introduction}

In quantum mechanics, it is a standard practice to perceive the Planck constant, $\hbar$, as a small parameter of the semiclassical approximation [1,2] and to write $\hbar \rightarrow 0$. One should keep in mind that, in principle, the symbol, $\hbar$, denotes a fundamental constant, which has a nontrivial physical dimension:

$$
\hbar=1.0545716 \times 10^{-27} \mathrm{erg} \cdot \mathrm{s}
$$

The smallness of $\hbar$ must be perceived in comparison to the "classical action", i.e., to the typical values of the classical action variables in the Hamilton-Jacobi formalism.

In statistical thermodynamics, the Boltzmann constant:

$$
k_{B}=1.3806488 \times 10^{-16} \mathrm{erg} \cdot \mathrm{K}^{-1}
$$


is a natural candidate for a similar role. The notation, $k_{B} \rightarrow 0$, looks just like $\hbar \rightarrow 0$, but it is relatively uncommon. Nonetheless, it can be defined in such a way that the corresponding limit transition is equivalent to $N \rightarrow \infty$, the large number of particles limit restricted by the condition that the values of specific extensive quantities (e.g., average energy per particle) remain fixed. One may compare this to an equivalent point of view on $\hbar \rightarrow 0$, which is also termed the "large quantum numbers" limit in quantum mechanics.

It is convenient to separate three levels of description of an abstract mechanical system:

L1: Classical mechanics;

L2: Semiclassical mechanics;

L3: Quantum mechanics.

In thermodynamics, there are also three levels:

11: Phenomenological thermodynamics;

12: Quasithermodynamics;

13: Statistical thermodynamics.

Quasithermodynamics is the theory of fluctuations of thermodynamic quantities if the number of particles in the system, $N \gg 1$, is large, but not "huge", and statistical thermodynamics is the microscopic theory of heat associated with such concepts as the Gibbs distribution and the constant, $k_{B}$. Phenomenological thermodynamics is described by the facts that the amount of a chemical substance in a system is measured in moles $\nu$ and not as a number of particles, $N$. The Boltzmann constant is not introduced into the theory yet, but the universal gas constant:

$$
R=8.3144621 \times 10^{7} \mathrm{erg} \cdot \mathrm{K}^{-1} \cdot \mathrm{mol}^{-1}
$$

is well defined.

It is a rather special coincidence that two so different theories, like classical mechanics and phenomenological thermodynamics, are closely related to such a fundamental mathematical concept, like the Lagrangian manifold. Moreover, a further analysis [3-5] leads to an idea of axiomatizing the asymptotic expansions of the partition function in terms of the tunnel canonical operator [6,7].

Take an abstract phenomenological thermodynamic system with extensive coordinates $E=\left(E_{0}, E_{1}, \ldots, E_{d}\right)$. The entropy function $S=S(E)$ satisfies $S\left(\lambda E_{0}, \lambda E_{1}, \ldots, \lambda E_{d}\right)=\lambda S(E)$, for all $\lambda>0$. According to the first law of thermodynamics:

$$
d S(E)=\beta_{0} d E_{0}+\beta_{1} d E_{1}+\cdots+\beta_{d} d E_{d}
$$

where $\beta=\left(\beta_{0}, \beta_{1}, \ldots, \beta_{d}\right)$ is the collection of the corresponding intensive coordinates. For example, take a one component system with $\nu$ moles of chemical substance described by the coordinates, $E_{0}=\nu$, the internal energy, $E_{1}$, and the volume, $E_{2}, d=2$. Then, $\beta_{0}=-\mu / T, \beta_{1}=1 / T$, and $\beta_{2}=p / T$, where $\mu$ is chemical potential, $T$ is absolute temperature and $p$ is pressure.

Assume that $S=S(E)$ is a smooth function over a domain, $\mathcal{D} \subset \mathbb{R}^{d+1}(E)$, and consider a manifold, $\Lambda_{S} \subset \mathbb{R}^{2(d+1)}(\beta, E)$ :

$$
\Lambda_{S}=\left\{(\beta, E) \mid \beta_{j}=\partial S(E) / \partial E_{j}, E \in \mathcal{D}, j=0,1, \ldots, d\right\}
$$


Let $i: \Lambda_{S} \rightarrow \mathbb{R}^{2(d+1)}(\beta, E)$ be the canonical embedding, and put:

$$
\omega=\sum_{j=0}^{d} d \beta_{j} \wedge d E_{j}
$$

The two-form $\omega$ defines a symplectic structure on $\mathbb{R}^{2(d+1)}(\beta, E)$, and we have: $i^{*}(\omega)=0$, i.e., $\Lambda_{S}$ is a Lagrangian manifold with respect to $\omega$. Note that $\omega$ does not depend on $S$, and therefore, $\Lambda_{S}$ is a Lagrangian manifold for any $S$.

The Lagrangian manifold, $\Lambda_{S}$ in thermodynamics is connected and simply connected; plus, there is a condition at infinity corresponding to the third law of thermodynamics. Furthermore, $\Lambda_{S}$ is covered by a single global chart with coordinates $E=\left(E_{0}, E_{1}, \ldots, E_{d}\right)$. The states of the thermodynamic equilibrium of the system are in one-to-one correspondence with the points of $\Lambda_{S}$, and it is convenient to lift $S=S(E)$ to a function $\widehat{S}=\widehat{S}(\alpha)$ on the Lagrangian manifold, $\alpha \in \Lambda_{S}$, so that $d \widehat{S}=i^{*}\left(\sum_{j=0}^{d} \beta_{j} d E_{j}\right)$. If we perceive $E_{j}, j=0,1, \ldots, d$ as an analogue of generalized coordinates in semiclassical mechanics, then the entropy is an action on the Lagrangian manifold of the equilibrium states of a thermodynamic system in the $E$-chart.

Consider now an abstract mechanical system with $D$ degrees of freedom described by coordinates $q=\left(q_{1}, q_{2}, \ldots, q_{D}\right)$ and the canonically conjugate momenta $p=\left(p_{1}, p_{2}, \ldots, p_{D}\right)$ corresponding to the symplectic structure $\omega_{\mathrm{cl}}=\sum_{i=1}^{D} d p_{i} \wedge d q_{i}$ on the phase space $\mathbb{R}^{2 D}(p, q)$. Take a Lagrangian manifold, $\Lambda_{\mathrm{cl}} \subset \mathbb{R}^{2 D}(p, q)$, and assume that $U \subset \Lambda_{\mathrm{cl}}$ is a $q$-chart on $\Lambda_{\mathrm{cl}}$. In semiclassical mechanics, the action $S_{\mathrm{cl}}=S_{\mathrm{cl}}(q)$ in $U$ has another important interpretation: it corresponds to the phase of the fast oscillating exponent in the Wentzel-Kramers-Brillouin (WKB) ansatz for the wavefunction, $\psi_{\hbar}(q)$, in the Schrödinger equation, $\psi_{\hbar}(q) \sim \exp \left(\mathrm{i} S_{\mathrm{cl}}(q) / \hbar\right)$. It is natural to expect that the semiclassical $\hbar \rightarrow 0$ methods of quantum mechanics can be "transplanted" to a thermodynamic Lagrangian manifold, $\Lambda_{S}$, and that this might lead to some new insights about statistical thermodynamics.

There exists a certain similarity between the quasithermodynamic fluctuation theory and the Heisenberg uncertainty relation, which has attracted the attention of many researchers [8-15]. This fact has already been known to N. Bohr and W. Heisenberg, who have tried to extend the philosophical concept of complementarity to thermodynamics. The corresponding analogy is far from being straightforward, since it depends strongly on the interpretation of the nature of intensive thermodynamic quantities. In [16-18], it is suggested to "span" a Pauli problem [19,20] over the fluctuations of thermodynamic quantities considered in the quasithermodynamic approximation. The similarities in the mathematical formalism of the classical mechanics and phenomenological thermodynamics are quite of interest from the perspective of relativistic quantum physics [21-25], as well as in "quantum thermodynamics" in the sense of [26-29]. There are also attempts to extend the analogy with quantum mechanics to non-equilibrium thermodynamics [30-35]. It is worth mentioning that numerical simulations of non-equilibrium multiparticle systems on a computer admit a natural parallelization [36].

The present work focuses on the equilibrium case. In quasithermodynamics, the intensive quantities and the specific extensive quantities enter the theory in a very symmetric way. We consider a problem of extension of this symmetry to statistical thermodynamics. The basic idea is to perceive the fluctuations of intensive quantities as fluctuations of specific extensive quantities in another auxiliary system. We provide a model example of thermodynamic duality and then state a generalized Pauli problem. 
It turns out that $2 k_{B} / N$, where $N$ is the number of particles in the model system, plays the same role as $\hbar$ in quantum mechanics.

\section{Quasithermodynamics and Quantization}

The Planck constant, $\hbar$, is similar to the Boltzmann constant, $k_{B}$, but there is an essential difference. In semiclassical mechanics, it enters the Bohr-Sommerfeld quantization condition, which for an abstract $D$-dimensional mechanical system on a Lagrangian torus, $\Lambda_{\mathrm{cl}} \subset \mathbb{R}^{2 D}(p, q)$, modulo the correction given by the Maslov index, is of the shape:

$$
\frac{1}{2 \pi} \oint_{\gamma_{k}} \sum_{j=1}^{D} p_{j} d q_{j} \sim \hbar n_{k}
$$

where $n_{k} \in \mathbb{Z}_{\geqslant 0}, k=1,2, \ldots, D$ and $\gamma_{k}$ correspond to the generators of the fundamental group, $\pi_{1}\left(\Lambda_{\mathrm{cl}}\right) \approx \mathbb{Z}^{D}$. For example, for a one-dimensional harmonic oscillator of frequency $\omega$ considered near a classical value of energy, $E$, we have: $E \omega^{-1} \sim \hbar n, n \in \mathbb{Z}, n \gg 1$. Intuitively, quantization is a splitting of the classical quantity, $E \omega^{-1}$, into a product of a "very small" quantity, $\hbar$, and a "very large" quantity, $n$.

The Lagrangian manifold, $\Lambda_{\mathrm{cl}}$, in the Bohr-Sommerfeld formula is not simply connected, while the Lagrangian manifold in thermodynamics is simply connected. This fact follows directly from the axiomatics of phenomenological thermodynamics. For an abstract thermodynamic system, $\Lambda^{0} \subset \mathbb{R}^{2(d+1)}(\widetilde{\beta}, E)$, of dimension $d$, where $E=\left(E_{0}, E_{1}, \ldots, E_{d}\right)$ are the extensive coordinates and $\widetilde{\beta}=\left(\beta_{0}, \beta_{1}, \ldots, \beta_{d}\right)$ are the intensive coordinates, we have:

$$
\oint_{\gamma} \sum_{j=0}^{d} \beta_{j} d E_{j}=0
$$

for any closed path $\gamma \subset \Lambda^{0}$. Therefore, the formula $\nu R \sim k_{B} N$, where $\nu$ is the number of moles and $N$ is the number of particles of a chemical substance in the system, is not exactly similar to $E \omega^{-1} \sim \hbar n$.

We should also point out that, since the entropy $S=S(E)$ satisfies $S\left(\lambda E_{0}, \lambda E_{1}, \ldots, \lambda E_{d}\right)=\lambda S(E)$, for all $\lambda>0$, the Lagrangian manifold $\Lambda^{0}$ has an additional property: if $\alpha^{0} \in \Lambda^{0}$ has coordinates $E_{j}\left(\alpha^{0}\right)$, $\beta_{l}\left(\alpha^{0}\right), j, l=0,1, \ldots, d$, then for every $\lambda>0$, the manifold, $\Lambda^{0}$, also contains $\alpha_{\lambda}^{0} \in \Lambda^{0}$ with coordinates $E_{j}\left(\alpha_{\lambda}^{0}\right)=\lambda E_{j}\left(\alpha^{0}\right), \beta_{l}\left(\alpha_{\lambda}^{0}\right)=\beta_{l}\left(\alpha^{0}\right), j, l=0,1, \ldots, d$.

We may assume that $E_{0}>0$ on $\Lambda^{0}$. Then, it is convenient to introduce the specific extensive coordinates $\varepsilon_{j}=E_{j} / E_{0}, j=1,2, \ldots, d$ and the specific entropy $s=S / E_{0}, s=s(\varepsilon)$, $\varepsilon=\left(\varepsilon_{1}, \varepsilon_{2}, \ldots, \varepsilon_{d}\right)$. The Lagrangian manifold, $\Lambda^{0}$, corresponds to $\Lambda \subset \mathbb{R}^{2 d}(\beta, \varepsilon), \beta=\left(\beta_{1}, \beta_{2}, \ldots, \beta_{d}\right)$, which is a Lagrangian manifold with respect to the symplectic structure $\omega=\sum_{j=1}^{d} d \beta_{j} \wedge d \varepsilon_{j}$, and we have:

$$
i_{\Lambda}^{*}\left(d s-\sum_{j=1}^{d} \beta_{j} d \varepsilon_{j}\right)=0
$$

where $i_{\Lambda}: \Lambda \rightarrow \mathbb{R}^{2 d}(\beta, \varepsilon)$ is the canonical embedding.

The previous formula, Equation (9), is a formula of phenomenological thermodynamics. Let us now discuss what happens in quasithermodynamics. Let $d=1$, and assume that the thermodynamic system 
contains $\nu$ moles of a single chemical substance. Let $E_{0}=\nu R / k_{B} ; R$ is the universal gas constant Equation (3) and $k_{B}$ is the Boltzmann constant Equation (2). Let $E_{1}$ denote the internal energy. Then, $\beta_{0}=-\mu k_{B} /(R T)$, and $\beta_{1}=1 / T$, where $\mu$ is the chemical potential and $T$ is the absolute temperature. It is convenient to write just $\beta$ and $\varepsilon$ in place of $\beta_{1}$ and $\varepsilon_{1}=E_{1} / E_{0}$, respectively.

The quantity, $\varepsilon$, is termed the specific internal energy of the system. Assume that $s^{\prime \prime}(\varepsilon)<0$ for all values of $\varepsilon$. Let $\phi(\beta)$ denote the Legendre transform of $s(\varepsilon)$ :

$$
\phi(\beta)=\left.(-\beta \varepsilon+s(\varepsilon))\right|_{\varepsilon=\varepsilon(\beta)}
$$

where $\varepsilon=\varepsilon(\beta)$ is the solution of the equation $-\beta+s^{\prime}(\varepsilon)=0$ with respect to $\varepsilon$. In quasithermodynamics, there is a large parameter in the formulae $N \rightarrow+\infty$, which corresponds to $\nu R / k_{B} \in \mathbb{R}$. More precisely, the scheme of a computation is as follows: find an asymptotic expansion with respect to $N \rightarrow+\infty$; keep the required number of terms, and then replace $N$ with a numerical value, $\nu R / k_{B}$. Take a point, $\alpha \in \Lambda$, and denote its coordinates in the ambient space, $\mathbb{R}^{2}(\beta, \varepsilon)$, as $(\beta(\alpha), \varepsilon(\alpha))$. The corresponding fluctuations, $\delta \beta$ and $\delta \varepsilon$, around the values $\beta=\beta(\alpha)$ and $\varepsilon=\varepsilon(\alpha)$, are described by the probability densities, $f_{\delta \beta}(y ; \alpha, N), y \in \mathbb{R}$, and $f_{\delta \varepsilon}(x ; \alpha, N), x \in \mathbb{R}$, which are approximated by the normal distributions of the shape:

$$
\begin{gathered}
f_{\delta \varepsilon}(x ; \alpha, N) \simeq\left(\frac{2 \pi k_{B}}{N \lambda}\right)^{1 / 2} \exp \left\{-N \frac{\lambda x^{2}}{2 k_{B}}\right\} \\
f_{\delta \beta}(y ; \alpha, N) \simeq\left(\frac{2 \pi k_{B} \lambda}{N}\right)^{1 / 2} \exp \left\{-N \frac{y^{2}}{2 k_{B} \lambda}\right\}
\end{gathered}
$$

where $\lambda=\lambda(\alpha)=-s^{\prime \prime}(\varepsilon(\alpha))=1 / \phi^{\prime \prime}(\beta(\alpha)), \alpha \in \Lambda, N \in \mathbb{R}$.

The interpretation of the fluctuations, $\delta \varepsilon$, is quite straightforward from the perspective of the canonical Gibbs formalism, which we discuss in more detail in the next section. On the other hand, an interpretation of the fluctuations of the inverse temperature, $\delta \beta$, turns out to be quite problematic. Moreover, there are different points of views on this subject, some of them described in [12].

Naively, if one accepts a "definition" of the inverse temperature as the parameter, $\beta$, in the canonical Gibbs distribution, then the fluctuations of $\delta \beta$ do not exist at all if the system is placed in a thermostat. At the same time, there is no problem to consider the quantities like the variance of energy in the system or the higher moments, so the fluctuations, $\delta \varepsilon$, receive a natural interpretation. What is then the meaning of the probability density Equation (12)?

In Landau-Lifshitz [37], the problem with $\delta \beta$ is essentially "swept under the carpet". They denote the fluctuation of inverse temperature as $\Delta \beta$ and the fluctuation of the inner energy as $\Delta E$ and put "by definition" $\Delta \beta \simeq\left(\partial^{2} S / \partial E^{2}\right)_{\nu} \Delta E$, where $S$ is the entropy as a function of the internal energy, $E$, and the number of moles, $\nu$, of the chemical substance. Basically, there is only one independent random variable, $\Delta E$, associated with a selected equilibrium state $(E, \nu)$ of the system, and $\Delta \beta$ is just a transformation of $\Delta E$. In the geometric picture involving the Lagrangian manifold, $\Lambda \subset \mathbb{R}^{2}(\beta, \varepsilon)$, one may then intuitively think of the fluctuations as follows: there is a point on $\Lambda$ that randomly moves a little around a fixed position, but it stays always on the manifold, i.e., the value of $\beta$ is immediately adjusted to the value of $\varepsilon$.

The Equations (11) and (12), imply that:

$$
\left\langle\left(N^{1 / 2} \delta \varepsilon\right)^{2}\right\rangle_{N, \alpha}\left\langle\left(N^{1 / 2} \delta \beta\right)^{2}\right\rangle_{N, \alpha} \simeq k_{B}^{2}
$$


where $\langle-\rangle_{N, \alpha}$ denotes taking the average with respect to the distributions in Equations (11) and (12). This is formally similar to the Heisenberg uncertainty relation in quantum mechanics, and we notice that $\hbar$ corresponds to the doubled Boltzmann constant, $2 k_{B}$. At the same time, if we accept the LandauLifshitz point of view, then the linear correlation coefficient between $N^{1 / 2} \delta \beta$ and $N^{1 / 2} \delta \varepsilon$ is equal to -1 . Take an abstract one-dimensional quantum system with a coordinate $\widehat{q}=x$ and the canonically conjugate momentum $\widehat{p}=-\mathrm{i} \hbar \partial / \partial x$, in a coherent state with a wavefunction :

$$
\psi_{\hbar}\left(x ; p_{0}, q_{0}, \lambda\right)=\left(\frac{2 \pi \hbar}{\lambda}\right)^{1 / 4} \exp \left(\mathrm{i} p_{0} x / \hbar\right) \exp \left\{-\frac{\lambda\left(x-q_{0}\right)^{2}}{4 \hbar}\right\}
$$

where $p_{0}, q_{0} \in \mathbb{R}$ and $\lambda>0$ are parameters. The corresponding quantum averages are $\langle\widehat{q}\rangle_{\hbar}=q_{0}$ and $\langle\widehat{p}\rangle_{\hbar}=p_{0}$, and for the fluctuations, $\delta \widehat{q}=\widehat{q}-q_{0}$ and $\delta \widehat{p}=\widehat{p}-p_{0}$; we have: $\left\langle(\delta \widehat{q})^{2}\right\rangle_{\hbar}\left\langle(\delta \widehat{p})^{2}\right\rangle_{\hbar}=\hbar^{2} / 4$, which is reminiscent of Equation (13). On the other hand, the linear correlation coefficient $\langle(\delta \widehat{q} \delta \widehat{p}+\delta \widehat{p} \delta \widehat{q}) / 2\rangle_{\hbar}\left\langle(\delta \widehat{q})^{2}\right\rangle_{\hbar}^{-1 / 2}\left\langle(\delta \widehat{p})^{2}\right\rangle_{\hbar}^{-1 / 2}$ between $\delta \widehat{q}$ and $\delta \widehat{p}$ is equal to zero and not to -1 .

Compare this to the following. In classical mechanics, we have a concept of an "action as a function of coordinates". To simplify the discussion, take a one-dimensional harmonic oscillator with the Hamiltonian $H(p, q)=\left(p^{2}+q^{2}\right) / 2$, and look at the isoenergetic surface $L=\left\{(p, q) \in \mathbb{R}^{2} \mid H(p, q)=c\right\}$, where $c>0$ is a parameter. $L$ is a one-dimensional Lagrangian manifold with respect to $d p \wedge d q$, and in a $q$-chart $U \subset L$, it can be described as $U=\{(p, q) \mid p=\partial S(q) / \partial q\}$, where $S(q)$ is the action as a function of $q, d S=p d q$ on $L$. If $\alpha \in L$ is a point representing the state of the system at time $t=0$, then after a short interval of time $\Delta t$, the system moves from $q(\alpha)$ to $q(\alpha)+\Delta q$, but one may say that the momentum, $p$, "immediately adjusts" its value from $p(\alpha)$ to $p(\alpha)+\Delta p$, so that the point stays on $L$. In the linear approximation: $\Delta p \simeq S^{\prime \prime}(q(\alpha)) \Delta q$. This equation on its own does not prevent us from studying the quantum mechanics of our system. One may construct, for example, a semiclassical wavefunction of the shape:

$$
\Psi_{\hbar}(x)=\int_{L} d \sigma(\alpha) \varphi(\alpha) \psi_{\hbar}(x ; p(\alpha), q(\alpha), 1)
$$

where $d \sigma$ is the measure on $L$ induced by $d p d q$ and $\varphi$ is a complex smooth function on $L$ with a finite support. In short, $\Delta p \simeq S^{\prime \prime}(q(\alpha)) \Delta q$ is a classical equation, and, similarly, $\Delta \beta \simeq\left(\partial^{2} S / \partial E^{2}\right)_{\nu} \Delta E$ corresponds to the physics after the thermodynamic limit.

We can see that an analogy between Equation (13) and the Heisenberg uncertainty relation is not $a$ priori excluded, but it is far from being straightforward. Let us therefore clarify the concept of intensive quantities in thermodynamics as it is accepted in the present paper by considering the definition of inverse absolute temperature. I assume that one is well aware of what is a canonical Gibbs distribution. Naively, in the standard notation, the inverse absolute temperature is the parameter, $\beta$, in this distribution. In the opinion of the author, this is not a conceptually correct way to define temperature. My starting point of view on thermodynamics is expressed in the book of M. Planck "Treatise on Thermodynamics" [38]. One should (not just can) start with phenomenological thermodynamics and perceive temperature (along with other quantities, like pressure, volume, etc.) as an independently defined phenomenological concept. It is wrong to derive the concept of temperature from a statistical model (the Gibbs distribution). The Gibbs distribution is just a possible explanation and an interpretation of what we see in terms of an underlying multiparticle mechanical system. This is captured by the term "mechanical theory of heat" which was more common in the old days than now. 
Similarly, with the fluctuations, $\delta \beta$, the phenomenology is described by the Einstein formula in Equation (12), but the "mechanical theory" of such fluctuations is, in my opinion, quite fuzzy. The aim of the present paper is to change this state of affairs a little. It is worth mentioning that in [14], B. Mandelbrot derives a kind of uncertainty relation for statistical estimators of thermodynamic quantities. I do not go in this direction in the present paper, but I consider essentially the same problem: what is the mechanical theory beyond the Einstein formula for $\delta \beta$ ?

The only reasonable possibility that I see to get some insight about such a theory is to use symmetry. The Equations (11) and (12), for the fluctuations, $\delta \beta$ and $\delta \varepsilon$, are completely similar. For the fluctuations, $\delta \varepsilon$, we have a "better" theory given by the canonical Gibbs distribution at inverse absolute temperature $\beta(\alpha), \alpha \in \Lambda$, and for the fluctuations, $\delta \beta$, we do not have one. It is natural to try to restore this symmetry in a "better" theory. One may try to construct an auxiliary thermodynamic system similar to the one that we have and define the notation, $\Lambda^{\prime}, \varepsilon^{\prime}, \beta^{\prime}, N^{\prime}$, in a totally similar way to $\Lambda, \varepsilon, \beta, N$. The idea is as follows: for a fixed point, $\alpha \in \Lambda$, there is a point, $\alpha^{\prime} \in \Lambda^{\prime}$, such that:

$$
\left\langle\left(N^{1 / 2} \delta \beta\right)^{n}\right\rangle \text { “better theory”, } N, \alpha=\left\langle\left(\left(N^{\prime}\right)^{1 / 2} \delta \varepsilon^{\prime}\right)^{n}\right\rangle
$$

for $n=2,3,4, \ldots$, where $\langle-\rangle$ on the right-hand side corresponds to the averages computed using the canonical Gibbs distribution for $N^{\prime} \in \mathbb{Z}$ particles at the inverse temperature $\beta^{\prime}=\beta^{\prime}\left(\alpha^{\prime}\right)$.

\section{Duality of Fluctuations}

As implied by the previous section, the idea of a "quantum complementarity" for the fluctuations of thermodynamic quantities should be handled with care. For a recent discussion, see $[8,12,13]$. Let us analyze this problem in terms of the inverse absolute temperature, $\beta$, and specific internal energy $\varepsilon$. We keep the notation for the Lagrangian manifold: $\Lambda \subset \mathbb{R}^{2}(\beta, \varepsilon)$. Consider a pair of cases:

(i) The system is in a thermostat.

(ii) The system is adiabatically isolated from the environment.

Case (i) is describing a parameter, $\beta_{*}$, which is the inverse absolute temperature of the thermostat. For an $N$-particle system, we have a Gibbs distribution:

$$
w_{n}^{(N)}\left(\beta_{*}\right)=\frac{1}{Z_{N}\left(\beta_{*}\right)} \exp \left(-\beta_{*} u_{n}^{(N)} / k_{B}\right)
$$

where $n$ is labeling all possible quantum states of the system, $w_{n}^{(N)}\left(\beta_{*}\right)$ is the probability of finding the system in a state, $n, u_{n}^{(N)}$ is the energy of the system in this state and $Z_{N}\left(\beta_{*}\right)$ is the partition function. Assume that the spectrum is discrete, $n=0,1,2, \ldots$, and that $\min _{n} u_{n}^{(N)} \geqslant 0$ ). If we look at the system from a phenomenological level, then we say that the inverse absolute temperature of the system $\beta=\beta_{*}$. If we look from the level of statistical thermodynamics, then we perceive the energy present in the system as a random variable; denote it $\mathcal{E}_{N, \beta_{*}}$. The possible values of $\mathcal{E}_{N, \beta_{*}}$ are $\left\{u_{n}^{(N)}\right\}_{n}$, and the corresponding probability weights are $\left\{w_{n}^{(N)}\left(\beta_{*}\right)\right\}_{n}$. The quantity, $\mathcal{E}_{N, \beta_{*}}$, fluctuates around the value $\left\langle\mathcal{E}_{N, \beta_{*}}\right\rangle=-\left.k_{B}(\partial / \partial \beta) \log Z_{N}(\beta)\right|_{\beta=\beta_{*}}$, where $\langle-\rangle$ denotes the mathematical expectation, with a nontrivial variance $\operatorname{Var}\left(\mathcal{E}_{N, \beta_{*}}\right)=\left.\left(-k_{B} \partial / \partial \beta\right)^{2} \log Z_{N}(\beta)\right|_{\beta=\beta_{*}}$. In the quasithermodynamic approximation, we have:

$$
\left\langle\mathcal{E}_{N, \beta_{*}}\right\rangle=O(N), \quad \operatorname{Var}\left(\mathcal{E}_{N, \beta_{*}}\right)=O(N)
$$


where $N \rightarrow+\infty$. In the notation of the Equation (11), the point, $\alpha \in \Lambda$, is determined by $\beta(\alpha)=\beta_{*}$, $\varepsilon(\alpha)=\lim _{N \rightarrow+\infty}\left\langle\mathcal{E}_{N, \beta_{*}}\right\rangle / N$, the fluctuation, $\delta \varepsilon$ corresponds, to $\left(\mathcal{E}_{N, \beta_{*}}-\left\langle\mathcal{E}_{N, \beta_{*}}\right\rangle\right) / N, N \in \mathbb{Z}$ and:

$$
s(\varepsilon)=\lim _{N \rightarrow+\infty} N^{-1} S^{(s t a t)}(N, N \varepsilon)
$$

where $S^{(\text {stat })}=S^{(\text {stat })}(N, E)$ is the Legendre transform of the function $\Phi^{(s t a t)}(N, \beta):=k_{B} \log Z_{N}(\beta)$ in the variable, $\beta$ :

$$
S^{(\text {stat })}(N, E)=\left.\left(\Phi^{(\text {stat })}(N, \beta)+\beta E\right)\right|_{\beta=\beta(N, E)}
$$

where $\beta=\beta(N, E)$ is the solution of the equation $E=\partial \Phi^{(\text {stat })}(N, \beta) / \partial \beta$ with respect to $\beta$. It is assumed that the corresponding limits and the Legendre transform exist.

Case (ii) is intuitively "dual" to case (i). The parameter, $\beta_{*}$, is replaced by the internal energy of the system, $E_{*}$. It is quite important to stress that $E_{*}$ is defined at the level of phenomenological thermodynamics. In this sense, it has nothing to do with the quantum mechanical energy spectrum of the system, $\left\{u_{n}^{(N)}\right\}_{n}$, which is used in statistical thermodynamics. The parameter, $E_{*}$, is not a "selected" energy level, $u_{n_{0}}^{(N)} \in\left\{u_{n}^{(N)}\right\}_{n}$.

It is a standard practice in the textbooks to replace Equation (17) with the microcanonical Gibbs distribution:

$$
W_{n}^{(N)}\left(E_{*} ; \delta\right)=\frac{1}{\Gamma_{N}\left(E_{*} ; \delta\right)} \chi_{[-\delta / 2, \delta / 2]}\left(u_{n}^{(N)}-E_{*}\right)
$$

where $W_{n}^{(N)}\left(E_{*} ; \delta\right)$ are the probabilities of finding the system in the states, $n=0,1,2, \ldots, \delta>0$ is a small parameter, $\chi_{[-\delta / 2, \delta / 2]}$ is the indicator function of the segment, $[-\delta / 2, \delta / 2]$, and $\Gamma_{N}\left(E_{*} ; \delta\right)$ is the normalization constant termed the statistical weight. The problem with Equation (21) is that it contains an arbitrary parameter $\delta>0$, and one first needs to compute the asymptotic behavior of the system as $N \rightarrow+\infty$ in the thermodynamic limit and then take the limit, $\delta \rightarrow 0$.

The quasithermodynamic Equations (11) and (12) for the fluctuations, $\delta \varepsilon$ and $\delta \beta$, look very similar, while Equations (17) and (21) are completely different. It is natural, on the other hand, to expect that the symmetry between $\delta \varepsilon$ and $\delta \beta$ stems from a deeper level of statistical thermodynamics. Unfortunately, essentially, there is no theory of fluctuations of intensive thermodynamic parameters beyond the quasithermodynamic theory.

It would be nice to have something like $\widetilde{w}_{m} \sim \exp \left(-E_{*} b_{m} / k_{B}\right)$ for the probability, $\widetilde{w}_{m}$, of the inverse temperature in the system to have a value of $b_{m}, m=0,1,2, \ldots$ There is $n o$ distribution like this that is known for a generic thermodynamic system, but, at the same time, nobody has proven that it cannot exist.

It is nonetheless completely legal to state the problem as follows. We may assume, without loss of generality, that $\beta$ and $\varepsilon$ have the same physical units:

$$
[\beta]=[\varepsilon]=\left[k_{B}^{1 / 2}\right]
$$

Since $\beta$ is initially the inverse absolute temperature, we need to redefine it by multiplying by a constant factor, and similarly, we need to redefine the energy by dividing it by the same factor. The canonical Gibbs distribution in Equation (17) keeps its original shape. Imagine that we have another thermodynamic system of $N^{\prime}$ particles with an energy spectrum, $\left\{u_{n}^{\prime\left(N^{\prime}\right)}\right\}_{n}, n=0,1,2, \ldots$, and the rescaled inverse absolute temperature, $\beta^{\prime}$, such that $\left[\beta^{\prime}\right]=\left[k_{B}^{1 / 2}\right]$. Define $\mathcal{E}_{N^{\prime}, \beta^{\prime}}^{\prime}$ in analogy with $\mathcal{E}_{N, \beta}$, 
replacing $\beta$ with $\beta^{\prime}$ and $\left\{u_{n}^{(N)}\right\}_{n}$ with $\left\{u_{n}^{\prime\left(N^{\prime}\right)}\right\}_{n}, n=0,1,2, \ldots$ Let $\Lambda^{\prime} \subset \mathbb{R}\left(\beta^{\prime}, \varepsilon^{\prime}\right)$ be the Lagrangian manifold for the second system defined similar to $\Lambda \subset \mathbb{R}^{2}(\beta, \varepsilon)$. We state the problem as follows: adjust the state, $\alpha^{\prime} \in \Lambda^{\prime}$, and the parameters of the construction of the second system in such a way that the fluctuations of $\delta \varepsilon^{\prime}:=\left(\mathcal{E}_{N^{\prime}, \beta^{\prime}\left(\alpha^{\prime}\right)}^{\prime}-\left\langle\mathcal{E}_{N^{\prime}, \beta^{\prime}\left(\alpha^{\prime}\right)}^{\prime}\right\rangle\right) / N^{\prime}$ approximate the fluctuations of the inverse temperature in the first system as well as possible. If $\alpha \in \Lambda$ is a selected point on the Lagrangian manifold, $\Lambda \subset \mathbb{R}^{2}(\beta, \varepsilon)$, of the first system, then we are interested in the fluctuations, $\delta \beta$, around the value $\beta=\beta(\alpha)$, and the ultimate goal is:

$$
\left\langle(\delta \beta)^{n}\right\rangle=\left\langle\left(\delta \varepsilon^{\prime}\right)^{n}\right\rangle
$$

for $n=2,3,4, \ldots$, where the moments of $\delta \beta$ on the left-hand side are determined by experiment. If we restrict the range of possible values of $n$ as $n=2,3, \ldots, n_{0}$, then, assuming the auxiliary thermodynamic system contains many enough parameters, the tuning Equation (23) becomes possible.

Before working out some examples, let us comment on the notation, $k_{B} \rightarrow 0$, mentioned in the Introduction. Suppose we have an entropy of the system, $S^{(\text {stat })}\left(E_{0}, E_{1}, \ldots, E_{d}\right)$, in terms of the extensive coordinates $E=\left(E_{0}, E_{1}, \ldots, E_{d}\right)$, which we have computed from the canonical partition function. Speaking of a thermodynamic limit, we are interested in the asymptotic behavior of a function:

$$
S_{\lambda}^{(s t a t)}(E):=\lambda^{-1} S^{(s t a t)}\left(\lambda E_{0}, \lambda E_{1}, \ldots, \lambda E_{d}\right)
$$

where $\lambda \rightarrow+\infty$ is a dimensionless large parameter. The limit $S(E)=\lim _{\lambda \rightarrow+\infty} S_{\lambda}^{(s t a t)}(E)$ is the entropy in the phenomenological thermodynamics, and its gradient determines the Lagrangian manifold, $\Lambda^{0} \subset \mathbb{R}^{2(d+1)}(\widetilde{\beta}, E), \widetilde{\beta}=\left(\beta_{0}, \beta_{1}, \ldots, \beta_{d}\right)$. The scheme of the computations can be organized as follows:

(1) Put formally, $k_{B}=1$ (this trick is similar to $\hbar=c=1$ ).

(2) Compute the required number of terms of the asymptotic expansion of $S_{\lambda}^{(s t a t)}(E)$ as $\lambda \rightarrow \infty$.

(3) Specialize $\lambda=1$.

(4) Recover the Boltzmann constant, $k_{B}$, given by Equation (2) from the dimension analysis.

Since at Step (2), the notation, $k_{B}$, is not reserved, we may re-denote $\lambda=\left(k_{B}\right)^{-1}$ and speak of $k_{B} \rightarrow 0$. At Step (3), we then specialize $k_{B}=1$, making the symbol, $k_{B}$, unreserved again.

Example 1. Take a system of $N$ quantum harmonic oscillators with frequency $\omega$. Put $k_{B}=1$. If we shift the ground energy level of an oscillator to zero, then the partition function of a single oscillator at inverse temperature $\beta$ is of the shape:

$$
Z_{1}(\beta)=\sum_{n=0}^{\infty} \exp \left(-\beta \bar{\varepsilon}_{n}\right)=(1-\exp (-\beta a))^{-1}
$$

where $a=\hbar \omega, \bar{\varepsilon}_{n}=a n$. Note that the parameters, $\beta$ and $a$, enter the formula $Z_{1}(\beta)=$ $\sum_{n=0}^{\infty} \exp (-\beta a n)$ in the same way, and one can perceive it as a sum $\sum_{n=0}^{\infty} \exp \left(-a \bar{\beta}_{n}\right), \bar{\beta}_{n}=\beta n$. The partition function of $N$ oscillators is $Z_{N}(\beta)=\left(Z_{1}(\beta)\right)^{N}$, and the corresponding derivatives yield:

$$
\begin{gathered}
\left\langle\mathcal{E}_{N, \beta}\right\rangle=N a(\exp (\beta a)-1)^{-1} \\
\operatorname{Var}\left(\mathcal{E}_{N, \beta}\right)=N^{-1} \exp (\beta a)\left\langle\mathcal{E}_{N, \beta}\right\rangle^{2}
\end{gathered}
$$


The entropy function $S^{(s t a t)}=S^{(s t a t)}\left(E_{0}, E_{1}\right)$, where $E_{0}=N$ and $E_{1}=E$ is the internal energy, is of the shape:

$$
S^{(s t a t)}(N, E)=N\left\{-\log \frac{a N}{E}+\left(1+\frac{E}{a N}\right) \log \left(1+\frac{a N}{E}\right)\right\}
$$

The Lagrangian manifold, $\Lambda \subset \mathbb{R}^{2}(\beta, \varepsilon)$, corresponding to the phenomenological specific entropy $s(\varepsilon)=\lim _{N \rightarrow+\infty} N^{-1} S^{(s t a t)}(N, N \varepsilon)$ is described by an equation:

$$
\beta=a^{-1} \log (1+a / \varepsilon)
$$

The Boltzmann constant is recovered as: $\beta=k_{B} a^{-1} \log (1+a / \varepsilon)$

Proposition 1 Let $X$ be a system of $N$ quantum harmonic oscillators of frequency $\omega$ put in $a$ thermostat at inverse absolute temperature $\beta$. Then, there exists a system, $X^{\prime}$, of $N^{\prime}$ quantum harmonic oscillators of frequency $\omega^{\prime}$ and a value, $\beta^{\prime}$, of the inverse absolute temperature in $X^{\prime}$, such that the quasithermodynamic fluctuations, $\delta \beta$, in $X$ are described by the same probability density as the quasithermodynamic fluctuations of the specific internal energy, $\delta \varepsilon^{\prime}$, in $X^{\prime}$.

Proof. Put $k_{B}=1$. Denote $a=\hbar \omega, a^{\prime}=\hbar \omega^{\prime}$. We need to satisfy a condition:

$$
\operatorname{Var}\left(\mathcal{E}_{N, \beta} / N\right) \operatorname{Var}\left(\mathcal{E}_{N^{\prime}, \beta^{\prime}}^{\prime} / N^{\prime}\right)=1
$$

We have the unknowns $a^{\prime}, \beta^{\prime}, N^{\prime}$ and the parameters, $a, \beta, N$. Impose a condition:

$$
\left(\left\langle\mathcal{E}_{N, \beta}\right\rangle / N\right)\left(\left\langle\mathcal{E}_{N^{\prime}, \beta^{\prime}}^{\prime}\right\rangle / N^{\prime}\right)=\beta^{\prime} \beta
$$

and put $N^{\prime}=N$. Taking into account Example 1, we obtain a system of equations:

$$
\begin{gathered}
\frac{a}{\exp (\beta a)-1} \frac{a^{\prime}}{\exp \left(\beta^{\prime} a^{\prime}\right)-1}=\beta^{\prime} \beta \\
\left(\beta^{\prime} \beta\right)^{2} \exp (\beta a) \exp \left(\beta^{\prime} a^{\prime}\right)=1
\end{gathered}
$$

Using the second equation, we derive from the first equation:

$$
\frac{\beta^{\prime} a^{\prime}}{1-\exp \left(-\beta^{\prime} a^{\prime}\right)}=\frac{1-\exp (-\beta a)}{\beta a}
$$

The function $\varphi(z)=z /(1-\exp (-z)), z \in \mathbb{R}$ is a continuous and monotonic function, $\varphi(0)=1$, such that $\varphi(z) \rightarrow 0$, if $z \rightarrow-\infty$, and $\varphi(z) \rightarrow+\infty$, if $z \rightarrow+\infty$. Our equation is of the shape $\varphi\left(\beta^{\prime} a^{\prime}\right)=1 / \varphi(\beta a)$, so if $\beta a$ is known, then $\beta^{\prime} a^{\prime}$ is uniquely determined. Once we know $\beta, a$ and $\beta^{\prime} a^{\prime}$, we can compute $\beta^{\prime}$ as $\beta^{\prime}=\beta^{-1} \exp (-\beta a / 2) \exp \left(-\beta^{\prime} a^{\prime} / 2\right)$. In other words, for any $\beta>0$ and $a>0$, the system Equations (32) and (33) have a unique solution $\left(\beta^{\prime}, a^{\prime}\right)=\left(\beta_{0}^{\prime}, a_{0}^{\prime}\right)$ with respect to $\beta^{\prime}>0$ and $a^{\prime}>0$. The required system, $X^{\prime}$, is described by $a^{\prime}=a_{0}^{\prime}$ and $N^{\prime}=N$, and the corresponding inverse temperature is $\beta^{\prime}=\beta_{0}^{\prime}$.

Remark 1. The construction of $\left(X^{\prime}, \beta^{\prime}\right)$ is not uniquely determined. The additional Equation (31) can be chosen differently. Let, for example, $\left\langle\mathcal{E}_{N^{\prime}, \beta^{\prime}}^{\prime}\right\rangle / N^{\prime}=\beta$. Keeping $N^{\prime}=N$, one arrives at a system:

$$
\begin{gathered}
a^{\prime}\left(\exp \left(\beta^{\prime} a^{\prime}\right)-1\right)^{-1}=\beta, \\
\exp \left(\beta a+\beta^{\prime} a^{\prime}\right)\left(\frac{\beta a}{\exp (\beta a)-1}\right)^{2}=1
\end{gathered}
$$


It follows that $\beta^{\prime} a^{\prime}=2 \log (\sinh (\beta a / 2) /(\beta a / 2))$. Substituting this into the first equation, one computes $a^{\prime}$. The system Equations (35) and (36) have a unique solution with respect to $\left(a^{\prime}, \beta^{\prime}\right)$. On the other hand, it does not follow that $\left\langle\mathcal{E}_{N, \beta}\right\rangle / N=\beta^{\prime}$, so the option Equation (31) is more symmetric. $\diamond$

Let us say that $\left(X^{\prime}, \beta^{\prime}\right)$ satisfying Proposition 1 is quasithermodynamically dual to $(X, \beta)$. The quasithermodynamic fluctuations of intensive and specific extensive thermodynamic quantities Equations (11) and (12) switch their roles if we switch between $(X, \beta)$ and $\left(X^{\prime}, \beta^{\prime}\right)$.

\section{The Pauli Problem}

Let $X=X_{N}(a)$ be the thermodynamic system, $N$, oscillators of frequency $\omega=a / \hbar$ in a thermostat at inverse temperature $\beta$. Denote $\mathcal{E}_{N, \beta}^{(a)}$ as the random variable corresponding to the energy contained in $X=X_{N}(a)$ at inverse temperature $\beta$. We have already computed $\left\langle\mathcal{E}_{N, \beta}^{(a)}\right\rangle$ and $\operatorname{Var}\left(\mathcal{E}_{N, \beta}^{(a)}\right)$, but it is not difficult to find the higher order cumulants, as well:

$$
K_{n}\left[\mathcal{E}_{N, \beta}^{(a)}\right]:=\left(-\frac{\partial}{\partial \beta}\right)^{n} \log Z_{N}(\beta ; a)
$$

where $n=1,2,3, \ldots, Z_{N}(\beta ; a)$ is the partition function of the system $X=X_{N}(a)$, and we work in the system of units $k_{B}=1$. Let us remind that if $t \rightarrow 0$ is a small parameter, then, for any $n \geqslant 1$, it holds:

$$
1+\sum_{m=1}^{n} \frac{t^{m}}{m !}\left\langle\left(\mathcal{E}_{N, \beta}^{(a)}\right)^{m}\right\rangle=\exp \left(\sum_{m=1}^{n} \frac{t^{m}}{m !} K_{m}\left[\mathcal{E}_{N, \beta}^{(a)}\right]\right)+O\left(t^{n+1}\right)
$$

as long as the corresponding moments exist. Expanding the exponent into a power series, one may recompute the cumulants in terms of the moments, and vice versa.

Since $K_{n}\left[\mathcal{E}_{N, \beta}^{(a)}\right]=\left.N a^{n}(-\partial / \partial x)^{n-1}\left(e^{x}-1\right)^{-1}\right|_{x=\beta a}$, we obtain:

$$
K_{n}\left[\mathcal{E}_{N, \beta}^{(a)}\right]=N a^{n} \sum_{m=1}^{n} \frac{c(n, m)}{\left(e^{\beta a}-1\right)^{m}}
$$

where $c(n, 1)=1, c(n, n)=(n-1) !$ and the coefficients, $c(n, m)$, satisfy a recurrent equation:

$$
c(n+1, m)=m c(n, m)+(m-1) c(n, m-1)
$$

where $2 \leqslant m \leqslant n-1$, and $n=1,2,3, \ldots$. This equation emerges in number theory in connection with the Bernoulli polynomials. For every pair of positive integers, $m$ and $n$, look at the sum:

$$
S_{m}(n)=1^{m}+2^{m}+\cdots+n^{m}
$$

This sum is known to be a polynomial in $n$ :

$$
S_{m}(n)=\frac{1}{m+1} \sum_{k=0}^{m}\left(\begin{array}{c}
m+1 \\
k
\end{array}\right) B_{k} n^{m+1-k}
$$

where $B_{k}$ are the Bernoulli numbers, $x /\left(e^{x}-1\right)=\sum_{n=0}^{\infty} B_{n} x^{n} / n !$. Another way to write the sum $S_{m}(n)$ is as follows:

$$
S_{m}(n)=\sum_{k=0}^{n-1}\left(\begin{array}{c}
n \\
k+1
\end{array}\right) c(m, k)
$$


where $c(m, k)$ are the same coefficients as in Equation (39). It is possible to describe them explicitly:

$$
c(n, m)=\frac{1}{m} \sum_{k=0}^{m}(-1)^{m-k}\left(\begin{array}{l}
m \\
k
\end{array}\right) k^{n}
$$

for all $m=1,2, \ldots, n$, and $n=1,2,3, \ldots$

In Proposition 1, we have constructed another system $X^{\prime}$ of $N^{\prime}=N$ oscillators with frequency $\omega^{\prime}=a^{\prime} / \hbar$ at inverse temperature $\beta^{\prime}$. Consider the cumulants of the energy in this system $\mathcal{E}_{N^{\prime}, \beta^{\prime}}^{\left(a^{\prime}\right)}$ at inverse temperature $\beta^{\prime}$ :

$$
K_{n}\left[\mathcal{E}_{N^{\prime}, \beta^{\prime}}^{\left(a^{\prime}\right)}\right]:=\left(-\frac{\partial}{\partial \beta^{\prime}}\right)^{n} \log Z_{N^{\prime}}\left(\beta^{\prime} ; a^{\prime}\right)
$$

where $n=1,2,3, \ldots$ We know that $\left(X^{\prime}, \beta^{\prime}\right)$ and $(X, \beta)$ are quasithermodynamically dual, i.e.:

$$
K_{2}\left[\mathcal{E}_{N^{\prime}, \beta^{\prime}}^{\left(a^{\prime}\right)}\right] K_{2}\left[\mathcal{E}_{N, \beta}^{(a)}\right]=N^{\prime} N
$$

Let us imagine for a short while that $\left(X^{\prime}, \beta^{\prime}\right)$ and $(X, \beta)$ are not just quasithermodynamically, but "completely" thermodynamically dual, i.e., for every $n \geqslant 2$, the $n$-th cumulant of of the fluctuation, $\delta \beta$, of the inverse temperature in $X$ coincides with $K_{n}\left[\mathcal{E}_{N^{\prime}, \beta^{\prime}}^{\left(a^{\prime}\right)} / N^{\prime}\right]$. Equivalently, this implies that we know all moments $\left\langle(\delta \beta)^{n}\right\rangle, n=2,3,4, \ldots$ In addition to the knowledge of the moments, $\left\langle(\delta \varepsilon)^{n}\right\rangle$, $n \geqslant 2$, for the fluctuations of the specific internal energy:

$$
\delta \varepsilon=\left(\mathcal{E}_{N, \beta}^{(a)}-\left\langle\mathcal{E}_{N, \beta}^{(a)}\right\rangle\right) / N
$$

can we unite these data in a single mathematical object?

In quantum mechanics, the role of such an object is played by the wavefunction or, more generally, by the Wigner quasiprobability function. We wish to find $R_{N}(x, y) \in L^{1}\left(\mathbb{R}^{2}(x, y)\right)$, satisfying $\int_{\mathbb{R}^{2}} d x d y R_{N}(x, y)=1$, such that:

$$
\left\langle(\delta \varepsilon)^{n}\right\rangle=\int_{\mathbb{R}^{2}} d x d y x^{n} R_{N}(x, y), \quad\left\langle(\delta \beta)^{n}\right\rangle=\int_{\mathbb{R}^{2}} d x d y y^{n} R_{N}(x, y)
$$

for $n=1,2,3, \ldots$ We do not require $R_{N}(x, y)$ to be non-negative, but all that matters is that the integrals $R_{N}^{(1)}(x):=\int_{\mathbb{R}} d y R_{N}(x, y)$ and $R_{N}^{(2)}(y):=\int_{\mathbb{R}} d x R_{N}(x, y)$ can be perceived as usual probability densities.

It is natural to consider a truncated version of the system Equation (48) by requiring that these equalities hold only for $n \leqslant n_{0}$, where $n_{0}$ is a positive integer, termed the degree of truncation. Then, we can always construct probability densities $f_{\delta \varepsilon}^{\left(n_{0}\right)}(x), x \in \mathbb{R}$ and $f_{\delta \beta}^{\left(n_{0}\right)}(y), y \in \mathbb{R}$, which are non-negative smooth real functions, such that:

$$
\int_{\mathbb{R}} d x x^{n} f_{\delta \varepsilon}^{\left(n_{0}\right)}(x)=\left\langle(\delta \varepsilon)^{n}\right\rangle, \quad \int_{\mathbb{R}} d y y^{n} f_{\delta \beta}^{\left(n_{0}\right)}(y)=\left\langle(\delta \beta)^{n}\right\rangle
$$

where $n=1,2, \ldots, n_{0}$. The element, $R_{N}(x, y) \in L^{1}\left(\mathbb{R}^{2}(x, y)\right)$, should satisfy:

$$
f_{\delta \varepsilon}^{\left(n_{0}\right)}(x)=\int_{\mathbb{R}} d y R_{N}(x, y), \quad f_{\delta \beta}^{\left(n_{0}\right)}(y)=\int_{\mathbb{R}} d x R_{N}(x, y)
$$

and it is necessary to impose some additional conditions on $R_{N}(x, y)$ to make this problem non-trivial. 
Proposition 2 Assume that $R_{N}(x, y) \in L^{1}\left(\mathbb{R}^{2}(x, y)\right)$ satisfies the system Equation (50) and that it is a symbol of a Weyl pseudo-differential operator $\widehat{R}_{N}=R_{N}\left(x,-\mathrm{i} \varkappa_{N} \partial / \partial x\right)$ on $L^{2}(\mathbb{R}(x))$, where $\varkappa_{N}>0$ is a constant. If $\widehat{R}_{N}$ is an orthogonal projector onto a one-dimensional subspace, then $\varkappa_{N} \sim 2 k_{B} / N$, as $N \rightarrow \infty$.

Proof. The parameter, $\varkappa_{N}$, is an analogue of $\hbar$ in the Pauli problem in quantum mechanics. Let us remind, that in one-dimensional quantum mechanics, the standard Pauli problem is defined as a problem of a reconstruction of a wavefunction, $\psi_{\hbar}(z) \in L^{2}(\mathbb{R}(z))$, from the knowledge of $\left|\psi_{\hbar}(z)\right|^{2}$ and $\left|\widetilde{\psi}_{\hbar}(z)\right|^{2}$, where $\widetilde{\psi}_{\hbar}(z), y \in \mathbb{R}$, is the $\hbar$-Fourier transform of $\psi_{\hbar}(z)$. Since $\delta \beta$ and $\delta \varepsilon$ are fluctuations of thermodynamic quantities around a point, $\alpha \in \Lambda$, on the Lagrangian manifold, $\Lambda \subset \mathbb{R}^{2}(\beta, \varepsilon)$, describing the system, they are approximated as $N \rightarrow+\infty$ by the normal distributions, $\delta \varepsilon \sim$ $\mathcal{N}\left(0, k_{B} N^{-1}\left[-s^{\prime \prime}(\varepsilon(\alpha))\right]^{-1}\right)$ and $\delta \beta \sim \mathcal{N}\left(0, k_{B} N^{-1}\left[-s^{\prime \prime}(\varepsilon(\alpha))\right]\right)$, where $s=s(\varepsilon)$ is the specific entropy as a function of the specific internal energy, $\varepsilon$.

Take a one-dimensional quantum system with a coordinate $\widehat{q}=z$ and the corresponding momentum $\widehat{p}=-\mathrm{i} \hbar \partial / \partial z$, and consider a Pauli problem with the densities of distribution of the coordinate and momentum given by the normal distributions, $\mathcal{N}(0, \lambda \hbar / 2)$ and $\mathcal{N}(0, \hbar /(2 \lambda))$, respectively, where $\lambda>0$ is a parameter. The solution, $\rho_{\hbar}(q, p ; \lambda)$, is known to exist, and it is given by the Wigner quasiprobability function associated with the wavefunction of the coherent state Equation (14) concentrated in $p_{0}=0$, $q_{0}=0$. The operator $\widehat{\rho}_{\hbar}(\lambda)=\rho_{\hbar}(z,-\mathrm{i} \hbar \partial / \partial z ; \lambda)$ is a one-dimensional orthogonal projector on $L^{2}(\mathbb{R}(z))$.

If we formally replace the parameter, $\lambda$, with $\left[-s^{\prime \prime}(\varepsilon(\alpha))\right]^{-1}, q$ with the coordinate, $x$, corresponding to $\delta \varepsilon, p$ with the coordinate, $y$, corresponding to $\delta \beta$, and $\hbar$ with $2 k_{B} / N$, then we obtain a solution of the Pauli problem, $R_{N}^{(q u a s i)}(x, y)$, for the fluctuations described by Equations (11) and (12). The Weyl pseudo-differential operator $\widehat{R}_{N}^{(\text {quasi })}=R_{N}^{(\text {quasi })}\left(x,-\mathrm{i} 2 k_{B} N^{-1} \partial / \partial x\right)$ is a one-dimensional orthogonal projector on $L^{2}(\mathbb{R}(x))$. Comparing this to $\widehat{R}_{N}=R_{N}\left(x,-\mathrm{i} \varkappa_{N} \partial / \partial x\right)$, we conclude: $\varkappa_{N} \sim 2 k_{B} / N$, as $N \rightarrow \infty$.

Proposition 2 implies that if we are interested in the Pauli problem in thermodynamics, then the parameter $\varkappa=2 k_{B} / N$ plays a similar role to the semiclassical parameter, $\hbar \rightarrow 0$, in quantum mechanics. We have a self-adjoint operator on $L^{2}(\mathbb{R}(x))$ with a unit trace:

$$
\widehat{R}_{N}=R_{N}\left(x,-\mathrm{i} \frac{2 k_{B}}{N} \frac{\partial}{\partial x}\right)
$$

where $x$ and $-\mathrm{i} 2 k_{B} N^{-1} \partial / \partial x$ are Weyl ordered. There is an additional condition on $R_{N}(x, y)$. In the quasithermodynamic limit, $N \rightarrow \infty$, the symbol, $R_{N}(x, y)$, must be concentrated in the point $(x, y)=(0,0)$ :

$$
R_{N}(x, y) \sim \frac{N}{2 \pi k_{B}} \exp \left\{\frac{N\left(x^{2} s^{\prime \prime}(\varepsilon(\alpha))+y^{2}\left[s^{\prime \prime}(\varepsilon(\alpha))\right]^{-1}\right)}{2 k_{B}}\right\}
$$

where $\alpha \in \Lambda, s^{\prime \prime}(\varepsilon(\alpha))<0$.

Remark 2. It might seem that there is an essential difference between the fluctuations of $\beta$ and $\varepsilon$ in statistical thermodynamics and the fluctuations of $p$ and $q$ in quantum mechanics. In quantum mechanics, we have a freedom of choice of what to measure, $p$ or $q$, but at the same time, we cannot choose to measure both. If the variance of $p$ tends to zero, then the variance of $q$ tends to infinity, and vice versa, in accordance with the Heisenberg uncertainty relation. If we put a thermodynamic system, $X$, in a 
thermostat with inverse absolute temperature $\beta_{*}$, then we "know" the inverse absolute temperature in $X$, but at the same time, the variance of the fluctuation of $\varepsilon$ is finite.

In quantum mechanics, we should distinguish between two different stages: a preparation of an experiment, and an interpretation of the outcome of an experiment. These stages are separated by an act of measurement. A preparation is described in the language of classical mechanics (i.e., the conditions of experiment, the choice of a measuring device, etc.). An outcome is described in the language of quantum mechanics (i.e., the spectrum of an observable, quantum numbers, etc.).

We should perceive thermodynamics in a similar way. When we put the system, $X$, in a thermostat, we make a choice of a measuring device, i.e., we say that we are going to observe the fluctuations of $\varepsilon$. We speak of $\beta_{*}$ in the language of phenomenological thermodynamics, and we interpret the observations of the fluctuations of $\varepsilon$ in the language of statistical thermodynamics.

If we surround the system, $X$, with adiabatic walls, then we make a choice of another "measuring device". We say that we are going to observe the fluctuations of $\beta$. The result of our preparation for the experiment is the internal energy, $E_{*}$, for which we use the language of phenomenological thermodynamics. The fluctuations of $\beta$ are observed in terms of statistical thermodynamics.

We cannot observe the fluctuations of $\varepsilon$ and $\beta$ both at the same time, just like we cannot do it with $p$ and $q$ in quantum mechanics. From the perspective of statistical thermodynamics, the symbols, $\beta_{*}$ and $E_{*}$, are parameters of distributions describing observable quantities, like the fluctuations of $\beta$ and $\varepsilon$. They are not "observables" themselves. What we can do is not measuring, but constructing statistical estimators for $\beta_{*}$ or $E_{*}$ working with finite samples of measurement outcomes.

One can find a much more detailed analysis of complementarity in statistical physics from a similar perspective in [8]. In the terminology of that paper, it is important to distinguish between the variables that parametrize the existence of the system (e.g., the mechanical macroscopic observables), and the conjugated variables that are relevant for dynamical descriptions of a tendency of the system to approach a thermodynamic equilibrium.

Remark 3. In principle, in thermodynamics, the number of particles, $N$, is large. On the other hand, we can formally specialize $N=1$ in the final formulae, and this leaves us with a rather "weird" entity: a thermodynamic system consisting of just one particle. At the same time, this particle is still a mechanical particle, so it has some mechanical coordinates, $q$, and momenta, $p$, described by quantum mechanics. It follows that we arrive at some kind of hybrid object: it is a quantum particle equipped with additional degrees of freedom of a thermodynamic nature. There is, for example, an additional degree of freedom, $\beta$, which is the inverse absolute "temperature" of the particle. Let us term this $N=1$ thermodynamic system a thermoparticle.

In thermodynamics, when one performs an intellectual leap from the phenomenological thermodynamics to the Gibbs distribution, one splits the system into a huge number of quantum particles, $\nu R=k_{B} N$; $\nu$ is the number of moles of the chemical substance of the system. Intuitively, it might be better to split the phenomenological thermodynamic system not into purely mechanical particles, but into thermoparticles. This is a "natural speculation" that goes far beyond the scope of the present article. 
Let us now briefly consider from the perspective of the Pauli problem a possible construction of the fluctuation theory for $\delta \varepsilon, \delta \beta$, beyond the Gaussian approximation in Equations (11), (12) and (52). Take a one-dimensional quantum mechanical system with a coordinate $\widehat{q}=x$ and the corresponding momentum $\widehat{p}=-\mathrm{i} \hbar \partial / \partial x$ acting on the Hilbert space, $L^{2}(\mathbb{R}(x))$. The usual Pauli problem deals with a reconstruction of a wavefunction, $\psi_{\hbar}(x) \in L^{2}(\mathbb{R}(x))$, from the knowledge of $\left|\psi_{\hbar}(x)\right|^{2}$ and $\left|\widetilde{\psi}_{\hbar}(p)\right|^{2}$, where:

$$
\widetilde{\psi}_{\hbar}(p):=\left(2 \pi e^{\mathrm{i} \pi / 2} \hbar\right)^{-1 / 2} \int_{\mathbb{R}} d x \exp (-\mathrm{i} p x / \hbar) \psi_{\hbar}(x)
$$

is the $\hbar$-Fourier transform of $\psi_{\hbar}(x)$.

Suppose now that the state of the system is not necessarily pure, but is described by a density matrix, $\rho_{\hbar}\left(x, x^{\prime}\right)$. The corresponding Wigner quasiprobability function, $\mathcal{W}_{\hbar}(p, q)$, is computed as follows:

$$
\mathcal{W}_{\hbar}(p, q)=\int_{\mathbb{R}} d x \rho_{\hbar}(q+x / 2, q-x / 2) \exp (-\mathrm{i} p x / \hbar)
$$

The normalization condition $(2 \pi \hbar)^{-1} \int_{\mathbb{R}^{2}} d p d q \mathcal{W}_{\hbar}(p, q)=1$. If the state is $\psi_{\hbar}(x)$, then $\rho_{\hbar}\left(x, x^{\prime}\right)=$ $\psi_{\hbar}(x) \bar{\psi}_{\hbar}\left(x^{\prime}\right)$, where the bar denotes the complex conjugation. For the coherent state Equation (14), we have: $\mathcal{W}_{\hbar}(p, q)=2 \exp \left(-\lambda\left(q-q_{0}\right)^{2} /(2 \hbar)\right) \exp \left(-2\left(p-p_{0}\right)^{2} /(\lambda \hbar)\right)$. The value of an integral, $(2 \pi \hbar)^{-1} \int_{\mathbb{R}^{2}} d q d p \mathcal{W}_{\hbar}(p, q) q^{m} p^{n}$, where $m, n \in \mathbb{Z}_{+}$, yields an average corresponding to the Weyl ordering of the expression, $\widehat{q}^{m} \widehat{p}^{n}$; for example: $(2 \pi \hbar)^{-1} \int_{\mathbb{R}^{2}} d q d p \mathcal{W}_{\hbar}(p, q) q p=\langle(\widehat{q} \widehat{p}+\widehat{p} \widehat{q}) / 2\rangle_{\hbar}$. Note that $(2 \pi \hbar)^{-1} \mathcal{W}_{\hbar}(p, q)$, considered as a replacement of the joint probability distribution density for the coordinate and the canonically conjugate momentum in classical mechanics, need not be non-negative, but it is real. Look at the observables:

$$
\widehat{Z}(\mu, \nu)=\mu \widehat{q}+\nu \widehat{p}
$$

where $\mu$ and $\nu$ vary over $\mathbb{R}$. Denote $\mathcal{T}(z ; \mu, \nu)$ as the density of the probability that $\widehat{Z}(\mu, \nu)$ has a value in $(z, z+d z]$. There exists a tomographic reconstruction formula [19,20]:

$$
\mathcal{W}_{\hbar}(p, q)=\frac{\hbar}{2 \pi} \int_{\mathbb{R}^{3}} \mathcal{T}(z ; \mu, \nu) \exp \{-\mathrm{i}(z-\mu q-\nu p)\} d z d \mu d \nu
$$

Note that it suffices to have the data only for the observables:

$$
\widehat{Z}(\cos (\theta), \sin (\theta))=\cos (\theta) \widehat{q}+\sin (\theta) \widehat{p}
$$

where $\theta \in[0, \pi)$, since the following should hold:

$$
\mathcal{T}(z ; \lambda \mu, \lambda \nu)=|\lambda|^{-1} \mathcal{T}\left(\lambda^{-1} z ; \mu, \nu\right)
$$

for any $\lambda \neq 0$.

In principle, to step outside the Gaussian approximation in Equations (11) and (12), one can mimic the reconstruction formula in Equation (56). The problem here is not in whether or not one is ready to perceive $(\delta \varepsilon, \delta \beta)$ in analogy with quantum mechanics, but in the fact that we do not really use $\delta \xi=\mu \delta \varepsilon+\nu \delta \beta$. It is nonetheless possible to obtain the quantum mechanical type formulae in a consistent way as follows. 
Write the variances, $\left\langle(\delta \varepsilon)^{2}\right\rangle_{N, \alpha}$ and $\left\langle(\delta \beta)^{2}\right\rangle_{N, \alpha}$, corresponding to Equations (11) and (12), as follows [16-18]:

$$
\left\langle(\delta \varepsilon)^{2}\right\rangle_{N, \alpha}=\int_{\mathbb{R}} d x x^{2}\left|\varphi_{h}(x)\right|^{2}, \quad\left\langle(\delta \beta)^{2}\right\rangle_{N, \alpha}=\int_{\mathbb{R}} d y y^{2}\left|\widetilde{\varphi}_{h}(y)\right|^{2}
$$

where $h=2 k_{B} / N, \varphi_{h}(x)=(\pi h / \lambda)^{1 / 4} \exp \left(-\lambda x^{2} /(2 h)\right)$, and $\widetilde{\varphi}_{h}(y)$ is the $h$-Fourier transform of $\varphi_{h}(x)$. The thermodynamic "wavefunction", $\varphi_{h}(x)$, is just a square root of $f_{\delta \varepsilon}(x ; \alpha, N), \lambda=\lambda(\alpha), \alpha \in \Lambda$. Let us perceive the $h$-Fourier transform $\widetilde{\varphi}_{h}(y)$ as follows:

$$
\widetilde{\varphi}_{h}(y)=\int_{\mathbb{R}} d x G(y, x, \pi / 2) \varphi_{h}(x)
$$

where $G(y, x, t), t>0$, is the solution of the Cauchy problem:

$$
\begin{gathered}
\mathrm{i} \hbar \frac{\partial G}{\partial t}=\frac{1}{2}\left(-h^{2} \frac{\partial^{2}}{\partial x^{2}}+x^{2}\right) G \\
\left.G\right|_{t=0}=\delta(y-x)
\end{gathered}
$$

The function, $G(y, x, t)$, is known explicitly:

$$
G(y, x, t)=\left(2 \pi e^{\mathrm{i} \pi / 2} h \sin t\right)^{-1 / 2} \exp \left\{\frac{\mathrm{i}}{h}\left(\frac{\cot t}{2}\left(y^{2}+x^{2}\right)-\frac{y x}{\sin t}\right)\right\}
$$

Take a little more generic function than $\varphi_{h}(x)$ :

$$
\varphi_{h}\left(x ; \lambda, x_{0}, y_{0}\right)=(\pi h / \lambda)^{1 / 4} \exp \left\{\frac{\mathrm{i}}{h}\left[\frac{\mathrm{i} \lambda}{2}\left(x-x_{0}\right)^{2}+y_{0} x\right]\right\}
$$

where $x_{0}, y_{0} \in \mathbb{R}$ and $\lambda>0$ are parameters. It is straightforward to check, that for:

$$
\varphi_{h}^{t}\left(y ; \lambda, x_{0}, y_{0}\right):=\int_{\mathbb{R}} d x G(y, x, t) \varphi_{h}\left(x ; \lambda, x_{0}, y_{0}\right)
$$

we obtain:

$$
\left|\varphi_{h}^{t}\left(y ; \lambda, x_{0}, y_{0}\right)\right|^{2}=\left(\pi h / \lambda_{t}\right)^{1 / 2} \exp \left\{-\lambda_{t}\left(y-c_{t}\right)^{2} / h\right\}
$$

where: $c_{t}=x_{0} \cos t+y_{0} \sin t$, and $\lambda_{t}=\left(\lambda^{-1} \cos ^{2} t+\lambda \sin ^{2} t\right)^{-1}$. Hence:

$$
\begin{gathered}
\int_{\mathbb{R}} d x x\left|\varphi_{h}^{t}\left(x ; \lambda, x_{0}, y_{0}\right)\right|^{2}=c_{t}=x_{0} \cos t+y_{0} \sin t \\
\int_{\mathbb{R}} d x\left(x-c_{t}\right)^{2}\left|\varphi_{h}^{t}\left(x ; \lambda, x_{0}, y_{0}\right)\right|^{2}=k_{B} N^{-1}\left(\lambda^{-1} \cos ^{2} t+\lambda \sin ^{2} t\right)
\end{gathered}
$$

where we have substituted $h=2 k_{B} / N$.

Consider now, as in Example 1 and Proposition 1, a system $X=X_{N}(a)$ of $N$ oscillators of frequency $\omega=a / \hbar$ at inverse temperature $\beta$. The average $\bar{\varepsilon}$ of the specific internal energy, $\mathcal{E}_{N, \beta}^{(a)} / N$, and its variance, $\left\langle(\delta \varepsilon)^{2}\right\rangle, \delta \varepsilon:=\left(\mathcal{E}_{N, \beta}^{(a)}-\left\langle\mathcal{E}_{N, \beta}^{(a)}\right\rangle\right) / N$, which we compute using the canonical Gibbs formalism, are of the shape:

$$
\bar{\varepsilon}=a\left(\exp \left(\beta a / k_{B}\right)-1\right)^{-1} \quad\left\langle(\delta \varepsilon)^{2}\right\rangle=N^{-1} \exp \left(\beta a / k_{B}\right)(\bar{\varepsilon})^{2}
$$

Take another system, $X^{\prime}$, of $N^{\prime}=N$ oscillators with frequency $\omega^{\prime}=a^{\prime} / \hbar$ at inverse temperature $\beta^{\prime}$. Adjust the combination of parameters $\left(a^{\prime}, \beta^{\prime}\right)$ in such a way that $\left\langle(\delta \varepsilon)^{2}\right\rangle\left\langle\left(\delta \varepsilon^{\prime}\right)^{2}\right\rangle=k_{B}^{2} N^{-2}$, where $\delta \varepsilon^{\prime}$ is 
the fluctuation of specific internal energy in $X^{\prime}$. Construct now a "homotopy" from $X$ to $X^{\prime}$. Let $X_{t}$, $t \in \mathbb{R}$ be a system of $N$ oscillators of frequency $\omega_{t}=a_{t} / \hbar$ at inverse temperature $\beta_{t}$. Determine the parameters, $a_{t}$ and $\beta_{t}$, from the requirement:

$$
\begin{gathered}
\bar{\varepsilon}_{t}=\bar{\varepsilon} \cos t+\bar{\varepsilon}^{\prime} \sin t \\
\left\langle\left(\delta \varepsilon_{t}\right)^{2}\right\rangle=\left\langle(\delta \varepsilon)^{2}\right\rangle \cos ^{2} t+\left\langle\left(\delta \varepsilon^{\prime}\right)^{2}\right\rangle \sin ^{2} t
\end{gathered}
$$

where $\bar{\varepsilon}_{t}$ is the average specific internal energy in $\left(X_{t}, \beta_{t}\right)$. Substituting the expressions $\bar{\varepsilon}_{t}=a_{t}\left(\exp \left(\beta_{t} a_{t} / k_{B}\right)-1\right)^{-1}$ and $\left\langle\left(\delta \varepsilon_{t}\right)^{2}\right\rangle=N^{-1} \exp \left(\beta_{t} a_{t} / k_{B}\right)\left(\bar{\varepsilon}_{t}\right)^{2}$, we note that $N$ cancels out and that $a_{t}$ and $\beta_{t}$ are uniquely determined, for every $t \in \mathbb{R}$ and:

$$
\left.\left(a_{t}, \beta_{t}\right)\right|_{t=0}=(a, \beta),\left.\quad\left(a_{t}, \beta_{t}\right)\right|_{t=\pi / 2}=\left(a^{\prime}, \beta^{\prime}\right)
$$

The right-hand side of the Equation (39), if we substitute $\beta_{t}$ in place of $\beta$, and $a_{t}$ in place of $a$, determines the higher order cumulants $K_{n}\left[\mathcal{E}_{N, \beta_{t}}^{\left(a_{t}\right)}\right], n=3,4,5, \ldots$, of the energy, $\mathcal{E}_{N, \beta_{t}}^{\left(a_{t}\right)}$ in $\left(X_{t}, \beta_{t}\right)$. One can always recompute these cumulants into the moments $\left\langle\left(\delta \varepsilon_{t}\right)^{n}\right\rangle, n=2,3,4, \ldots$, if necessary, $\left\langle\delta \varepsilon_{t}\right\rangle=0$. Assume that for some $n_{0} \in \mathbb{Z}, n_{0} \geqslant 3$, we have constructed a function, $\mathcal{T}(z ; \mu, \nu)$, such that:

$$
\begin{gathered}
\mathcal{T}(z ; \lambda \cos t, \lambda \sin t)=|\lambda|^{-1} \mathcal{T}\left(\lambda^{-1} z ; \cos t, \sin t\right) \\
\left\langle\left(\delta \varepsilon_{t}\right)^{n}\right\rangle=\int_{\mathbb{R}} d z z^{n} \mathcal{T}(z ; \cos t, \sin t)
\end{gathered}
$$

where $\lambda \neq 0, t \in[0, \pi), n=1,2, \ldots, n_{0}$. Then, it remains to apply the tomographic reconstruction formula in Equation (56) replacing $\hbar$ with $2 k_{B} / N$. This yields a joint quasiprobability function describing $(\delta \varepsilon, \delta \beta)$, which is more advanced than the right-hand side of Equation (52).

One should stress that this function is obtained by a mathematical analogy, and it certainly requires experimental tests and a theoretical extension to more realistic systems. This can be a subject of future research.

\section{Conclusions}

In phenomenological thermodynamics, as well as in the theory of quasithermodynamic fluctuations, there exists a certain symmetry between intensive and extensive quantities. At the same time, this symmetry is not immediately visible in statistical thermodynamics, since the Gibbs formalism deals only with the fluctuations of extensive quantities (energy, number of particles, etc.). An interpretation of the fluctuations of intensive quantities is a rather controversial issue.

In quasithermodynamics, the probability densities for the fluctuations of specific extensive quantities (e.g., $\delta \varepsilon$, where $\varepsilon$ is specific internal energy) and the associated intensive quantities (e.g., $\delta \beta$, where $\beta$ is inverse absolute temperature) are completely similar. In particular, the corresponding variances satisfy the same asymptotic estimates, $\left\langle(\delta \varepsilon)^{2}\right\rangle=O\left(N^{-1}\right)$ and $\left\langle(\delta \beta)^{2}\right\rangle=O\left(N^{-1}\right)$, as the number of particles $N \rightarrow+\infty$. Mathematically, it is possible to perceive the quasithermodynamic fluctuations of intensive quantities in a system $X$, as quasithermodynamic fluctuations of specific extensive quantities in another system, $Y$.

In the present paper, it is suggested to extend this fact to statistical thermodynamics in order to construct a fluctuation theory of intensive quantities. This turns out to be possible for a model system of 
$N$ quantum harmonic oscillators of the same frequency if one takes into account an analogy between the transition from quantum to classical mechanics and the transition from statistical to phenomenological thermodynamics. In the main text, we "span" a generalized Pauli problem over the fluctuations, $\delta \beta$ and $\delta \varepsilon$, and apply the tomographic reconstruction formula. This yields a self-adjoint non-negative operator, $\widehat{R}$, with a unit trace on $L^{2}(\mathbb{R}(x))$ :

$$
\widehat{R}=R_{N}\left(x,-\mathrm{i} \frac{2 k_{B}}{N} \frac{\partial}{\partial x}\right)
$$

which is similar to the density matrix operator in quantum mechanics (we use the Weyl quantization). The symbol, $R_{N}(x, y)$, replaces the Wigner function, and it is concentrated in a point $(x, y)=(0,0)$ in the quasithermodynamic limit, $N \rightarrow+\infty$. The combination, $2 k_{B} / N$, plays the same role as the Planck constant, $\hbar$.

\section{Conflicts of Interest}

The author declares no conflict of interest.

\section{References}

1. Maslov, V.P.; Fedoriuk, M.V. Semi-Classical Approximation in Quantum Mechanics; Springer: Berlin, Germany, 1981; p. 316.

2. Leray, J. Lagrangian Analysis and Quantum Mechanics: A Mathematical Structure Related to Asymptotic Expansions and the Maslov Index; The MIT Press: Cambridge, MA, USA, 1982; p. 288.

3. Maslov, V.P. Geometric quantization of thermodynamics, phase transitions and asymptotics at critical points. Trans. Math. Notes 1994, 56, 984-985.

4. Maslov, V.P. Analytic extension of asymptotic formulas, and the axiomatics of thermodynamics and quasithermodynamics. Trans. Funct. Anal. Appl. 1994, 28, 247-256.

5. Maslov, V.P. Geometric "quantization" of thermodynamics, and statistical corrections at critical points. Trans. Theor. Math. Phys. 1994, 101, 1466-1472.

6. Maslov, V.P.; Nazaikinskii, V.E. The tunnel canonical operator in thermodynamics. Trans. Funct. Anal. Appl. 2006, 40, 173-187.

7. Maslov, V.P. Asymptotic Methods and Perturbation Theory; Nauka: Moscow, Russia, 1988; p. 311.

8. Velazquez Abad, L. Principles of classical statistical mechanics: A perspective from the notion of complementarity. Ann. Phys. 2012, 327, 1682-1693.

9. Kazinski, P.O. Stochastic deformation of a thermodynamic symplectic structure. Phys. Rev. E 2009, 79, doi:10.1103/PhysRevE.79.011105.

10. Lavenda, B.H. Thermodynamic uncertainty relations and irreversibility. Int. J. Theor. Phys. 1987, 26, 1069-1084.

11. Rudoi, Y.G.; Sukhanov, A.D. Thermodynamic fluctuations within the Gibbs and Einstein approaches. Phys. Usp. 2000, 43, 1169-1199.

12. Uffink, J.; van Lith, J. Thermodynamic uncertainty relations. Found. Phys. 1999, 29, 655-692. 
13. Velazquez, L.; Curilef, S. A thermodynamic fluctuation relation for temperature and energy. J. Phys. A 2009, 42, 095006.

14. Mandelbrot, B. The role of sufficiency and of estimation in thermodynamics. Ann. Math. Stat. 1962, 33, 1021-1038.

15. Ruppeiner, G. Riemannian geometry in thermodynamic fluctuation theory. Rev. Mod. Phys. 1995, 67, 605-659.

16. Ruuge, A.E. A tropical analogue of the Pauli problem and a splitting of thermodynamics. Dokl. Math. 2013, 88, 482-485;

17. Ruuge, A.E. An asymptotics of the Pauli problem in thermodynamics. Dokl. Math. 2013, 87, 360-363.

18. Ruuge, A.E. Pauli problem in thermodynamics. 2013, arxiv:1208.2919v2.

19. Ibort, A.; Man'ko, V.I.; Marmoc, G.; Simonic, A.; Ventrigliac, F. An introduction to the tomographic picture of quantum mechanics. Phys. Scr. 2009, 79, 065013.

20. Mancini, S.; Man'ko, V.I.; Tombesi, P. Classical-like description of quantum dynamics by means of symplectic tomography. Found. Phys. 1997, 27, 801-824.

21. Balian, R.; Valentin, P. Hamiltonian structure of thermodynamics with gauge. Eur. Phys. J. B Condens. Matter Phys. 2001, 21, 269-282.

22. Rovelli, C. Statistical mechanics of gravity and the thermodynamical origin of time. Class. Quantum Grav. 1993, 10, 1549-1566.

23. Connes, A.; Rovelli, C. Von Neumann algebra automorphisms and time-thermodynamics relation in generally covariant quantum theories. Class. Quantum Grav. 1994, 11, 2899-2917.

24. Montesinos, M.; Rovelli, C. Statistical mechanics of generally covariant quantum theories: A Boltzmann-like approach. Class. Quantum Grav. 2001, 18, 555-569.

25. Rajeev, S.G. Quantization of contact manifolds and thermodynamics. Ann. Phys. 2008, 323, 768-782.

26. Henrich, M.J.; Michel, M.; Mahler, G. Small quantum networks operating as quantum thermodynamic machines. Europhys. Lett. 2006, 76, 1057-1063.

27. Quan, H.T.; Liu, Y.-X.; Sun, C.P.; Nori, F. Quantum thermodynamic cycles and quantum heat engines. Phys. Rev. E 2007, 76, 031105.

28. Linden, N.; Popescu, S.; Short, A.J.; Winter, A. Quantum mechanical evolution towards thermal equilibrium. Phys. Rev. E 2009, 79, 061103.

29. Skrzypczyk, P.; Brunner, N.; Linden, N.; Popescu, S. The smallest refrigerators can reach maximal efficiency. J. Phys. A 2011, 44, 492002.

30. Onsager, L.; Machlup, S. Fluctuations and irreversible processes. Phys. Rev. 1953, 91, $1505-1512$.

31. Machlup, S.; Onsager, L. Fluctuations and irreversible processes. II. Systems with kinetic energy. Phys. Rev. 1953, 91, 1512-1515.

32. Mehrafarin, M. Canonical operator formulation of non-equilibrium thermodynamics. J. Phys. A 1993, 16, 5351-5363. 
33. Acosta, D.; Fernández de Córdoba, P.; Isidro, J.M.; Santander, J.L.G. Emergent quantum mechanics as a classical, irreversible thermodynamics. Int. J. Geom. Methods Mod. Phys. 2013, 10, 1350007 .

34. Fernández de Córdoba, P.; Isidro, J.M.; Perea, M.H. Emergent quantum mechanics as a thermal ensemble. 2013, arXiv:1304.6295v2.

35. Ruuge, A.E. Microlagrangian manifolds and quasithermodynamic fluctuations of nonequilibrium states. 2010, arXiv:1008.3297v2.

36. Inozemtseva, N.G.; Perepelkin, E.E.; Sadovnikov, B.I. Optimization of Algorithms in Mathematical Physics Problems for Graphics Processing Units; Physics Faculty of M. V. Lomonosov Moscow State University: Moscow, Russia, 2012.

37. Landau, L.D.; Lifshitz, E.M. Statistical Physics , 3rd ed.; Butterworth-Heinemann: Oxford, UK, 1980; Volume 5, p. 544.

38. Planck, M. Treatise on Thermodynamics, 3rd ed.; Dover Publications: New York, NY, USA, 2010; p. 320.

(c) 2013 by the author; licensee MDPI, Basel, Switzerland. This article is an open access article distributed under the terms and conditions of the Creative Commons Attribution license (http://creativecommons.org/licenses/by/3.0/). 